\title{
EFFECT OF POSTURAL CONTROL BIOMECHANICAL GAIN ON PSYCHOPHYSICAL DETECTION THRESHOLDS IN ANTERIOR HORIZONTAL TRANSLATION OF STANDING BLINDFOLDED SUBJECTS
}

\author{
Shahrokh N Sani ${ }^{1}$ and Charles J Robinson ${ }^{2}$ \\ ${ }^{1}$ Department of Computer Science, Morehead State University, Morehead KY, USA \\ ${ }^{2}$ Depts of Electrical \& Computer Engr, Clarkson University, Potsdam, NY, USA
}

\begin{abstract}
A Sliding Linear Investigative Platform for Assessing Lower Limb Stability (SLIP-FALLS) was employed to study postural control biomechanical reaction to external perturbations in a short $\leq 16 m m$ postural perturbation. Head acceleration were evaluated while blindfolded subjects stood on a platform that was given a short anterior perturbation presented in one of 2 sequential $4 s$ intervals (2-Alternative-ForcedChoice) for a set of 30 trials. Anterior-Posterior head acceleration (Head Accl AP) were investigated among the movement and non-movement intervals for the healthy adults. A strong ringing signal was observed in Head Accl AP movement interval that was absent in non-movement interval. A positive power law trading relationship was found between Head Accl AP gain and move length standing blindfolded subjects. This could explain the observed negative power law relationship between translation length and peak acceleration threshold in previous psychophysical detection threshold studies.
\end{abstract}

\section{KEYWORDS}

psychophysical - head acceleration- Postural control- external perturbation- frequency dependency

\section{INTRODUCTION}

The human balance control system uses different information sources to maintain the body's balance. Therefore, balance tests evaluate the postural control system by restricting one input or allowing all inputs. Postural control is studied from different aspects such as human biomechanics, human neurophysiology and psychophysiology of human balance. A human balance test could be a simple clinical test or a completely instrumented test that assesses various feedback control systems involved in the human balance control system. Human balance tests can be categorized into static and dynamic tests [1].

Dynamic balance tests observe the reaction of the subject's postural control to external perturbation. The external perturbation applies to the feet or ankle by translating or rotating the platform the subject stands on. Dynamic stability tests have been analyzed by many different measurements. Body center of pressure (COP), segment movements, and muscle activation (EMG) have been used to assess the reaction of the human balance system to various perturbations [2]. 
Detecting perturbations plays a significant role in preventing a slippage that can lead to a fall. The Sliding Platform for Assessing Lower Limb Stability with Synced Tracking, EMG and Pressure measurement (SLIP-FALLS-STEPm) system is a novel set of equipment for studying human balance control [3]. It utilizes a linear motor and air bearing slides to drive small translational horizontal movements $(0.25$ to $16 \mathrm{~mm})$ that are in the subject's natural sway range while the subject stands on the platform. The experiment is continued until the subject's detection acceleration threshold is reached or after 30 trials. It able us to study the psychophysical, biomechanical and neurophysiological responses to short horizontal perturbations that are at the edge of perception [3]. Our previous studies revealed that there exists a robust psychophysical negative power law trading relationship between translation length and peak acceleration threshold [4-6]. For example, a $1 \mathrm{~mm}$ translation required a geometrically higher Psychophysical acceleration threshold than a $16 \mathrm{~mm}$ translation.

Grant et al. showed that the frequency of perturbation acceleration has an effect on movement perception [7]. Benson et al. [8] observed a correlation between movement perception and both the peak acceleration and the peak jerk. They also showed there is a reverse relationship between frequency of the stimulus and detection threshold (e.g., detection threshold decreases with increasing frequency of the stimulus). Heerspink et al. [9] comprehensively investigated the relationship between stimulus frequency and detection threshold. They found that there is a reverse relationship between frequency of the stimulus and detection threshold for lower frequencies, and there is no relationship between frequency of the stimulus and detection threshold for higher frequency. A study by Grant and Haycock [10] reported that either increasing the acceleration or the jerk increases the movement perception threshold. A totally different behavior was reported by Mah et al. [11]. They reported non-jerk sensitivity behavior for low frequencies and a direct relationship between frequency of the stimulus and detection threshold for high frequencies (increase in frequency causes an increase in the threshold).

Subject's head acceleration reaction to external perturbation has been studied throughout the paper. Our results revealed that the postural control react to perturbation with producing a ringing signal in the head. A positive power law relationship between the anterior translation lengths and a subject's head acceleration gain was observed that could explain the reported negative power law relationship between translation length and peak acceleration threshold in previous psychophysical detection threshold studies [4-6].

\section{METHOD.}

\subsection{Participants and Test Protocols}

The study participants were 9 healthy adult subjects and without lower limb peripheral neuropathy. They were asked to stand on the transitional platform and were blindfolded during all trials. In each trial, a subject heard four verbal instructions from the headphone: "ready", "one", "two" and "decide". Each stimulus interval was 4s long. When the subject heard the word "decide", (s)he had to press the wireless doorbell button once or twice based on perceiving a movement in interval one or two. The length of the platform movement was $16 \mathrm{~mm}, 4 \mathrm{~mm}$, and $1 \mathrm{~mm}$. A set of 30 trials maximum was collected for each subject. The platform position and acceleration, the subject's anterior posterior and medial-lateral centers of pressure (APCOP and MLCOP), lower limb EMG signals and head acceleration in the $\mathrm{X}, \mathrm{Y}$ and $\mathrm{Z}$ directions were collected at $1000 \mathrm{~Hz}$ and also processed into engineering units and stored at $100 \mathrm{~Hz}$. 
A 2-Alternative Force Choice (2AFCi) psychophysical procedure was used to determine the acceleration threshold at displacements of 1,4 and $16 \mathrm{~mm}$. The experiment was continued until the subject's detection acceleration threshold or 30 trials was reached. Finding the subject's psychophysical threshold for the perception of a given displacement was the main goal during the 30 trials [3].The stimulus was given in interval 1 or 2 and the subject was compelled to choose by button press the interval in which (s)he perceived a movement. The platform acceleration value either increased (for non-detect) or decreased (for a pair or triad of detects) in the next trial based on a modified Parameter Estimation by Sequential Testing (PEST) paradigm [12].

\subsection{Biomechanical Response to 1,4 and $16 \mathrm{~mm}$ Perturbation}

A Matlab based GUI (Graphical User Interface) created to analyze the subjects' head acceleration data in adjustable movement and non-movement time interval length during 30 trials of $2 \mathrm{AFC}$ experiments. The designed software was able to adjust the movement and non-movement time interval length. In each trial, 15 to $19 \mathrm{~s}$ of head AP acceleration data were recorded by our sensor. For $1 \mathrm{~mm}$ and $4 \mathrm{~mm}$ displacement, the interval 1 movements usually start after $4 \mathrm{~s}$ and interval 2 movements after $8 \mathrm{~s}$. For a $16 \mathrm{~mm}$ displacement, interval 2 movements usually start at $10 \mathrm{~s}$. Fig. 1 shows platform position and acceleration (AP) in movement interval during thirty 1,4 and $16 \mathrm{~mm}$ 2AFC experimental displacements. The patch line plot has been used to show the moves over all 30 trials.

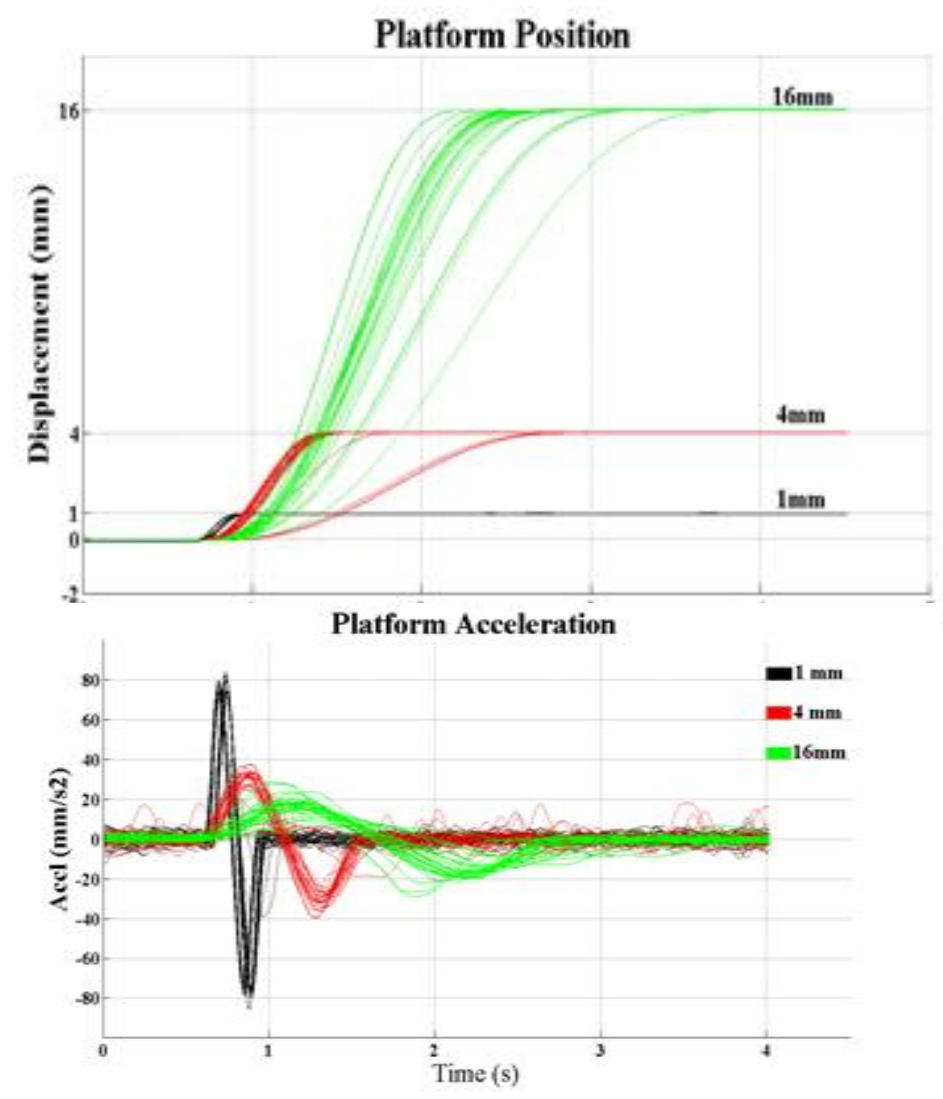

Fig. 1. Movement interval platform Position and acceleration patch lines during thirty $2 \mathrm{AFC}$ trials of 1,4 , and $16 \mathrm{~mm}$ translation 


\section{RESULTS}

\subsection{Anterior-Posterior Head Acceleration in Movement Interval}

The subject's biomedical response to perturbation can be neurophysiological (i.e., EMG) or biomechanical (e.g., APCOP or head acceleration). In this study we preferred to use the biomechanical response for our analysis. We performed this analysis by focusing on head acceleration in the anterior-posterior direction (AP) between the movement and non-movement interval. We consider Subject's head AP acceleration as only biomechanical response to the perturbations. A ringing signal was observed in movement interval that was absent in nonmovement interval. Head AP acceleration in movement intervals during 30 trials of the 1, 4 and $16 \mathrm{~mm} 2 \mathrm{AFC}$ experiments has been shown in Fig. 2.

\subsection{Observation of Gain}

We considered the point-by-point ensemble average of platform acceleration in 30 trials to be the input to our human postural system, and the ensemble averaged head AP acceleration as one output. Fig. 3.a shows the point by point average of the platform acceleration and head AP acceleration in 30 trials of the 1, 4 and 16mm 2AFC experiments in same plots. The amplitude of platform acceleration is shown by A1 and the amplitude of Head AP acceleration by A2 (see Fig. 3.b).Head AP gain is defined by dividing A2 by A1. Our designed GUI is able to calculate automatically the Gain (Gain = the amplitude of the input/amplitude of the output) for different displacements and subjects

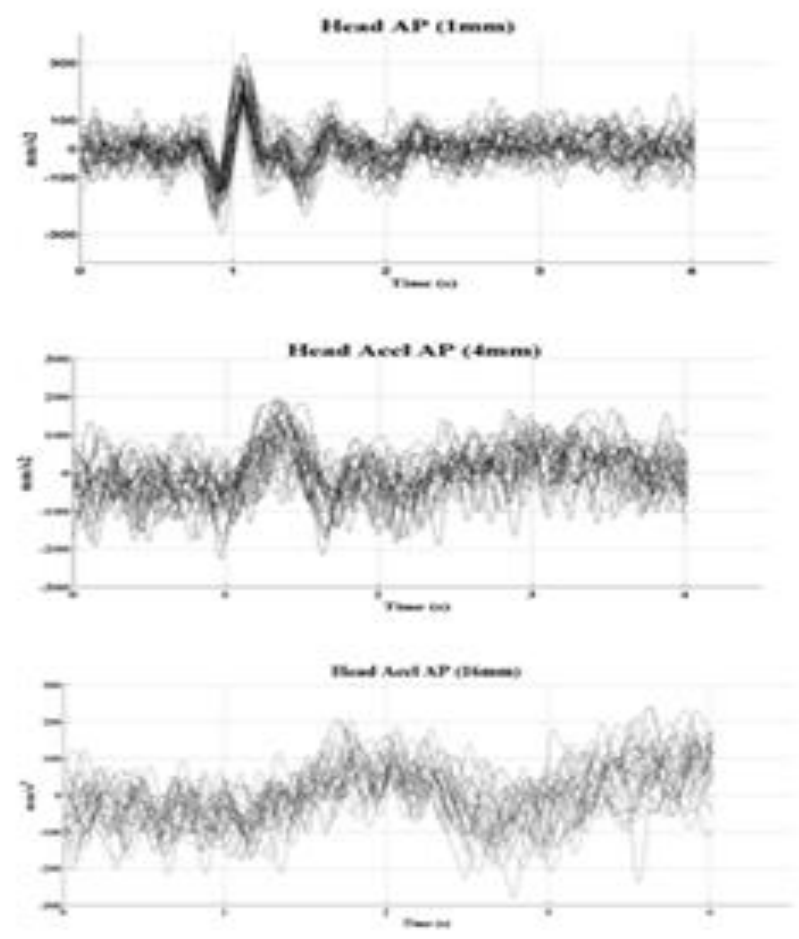

Fig. 2. Head AP acceleration patch lines in movement interval during thirty 2 AFC trials of 1 , 4, and $16 \mathrm{~mm}$ translations. 
International Journal on Bioinformatics \& Biosciences (IJBB) Vol.6, No.3/4, December 2016
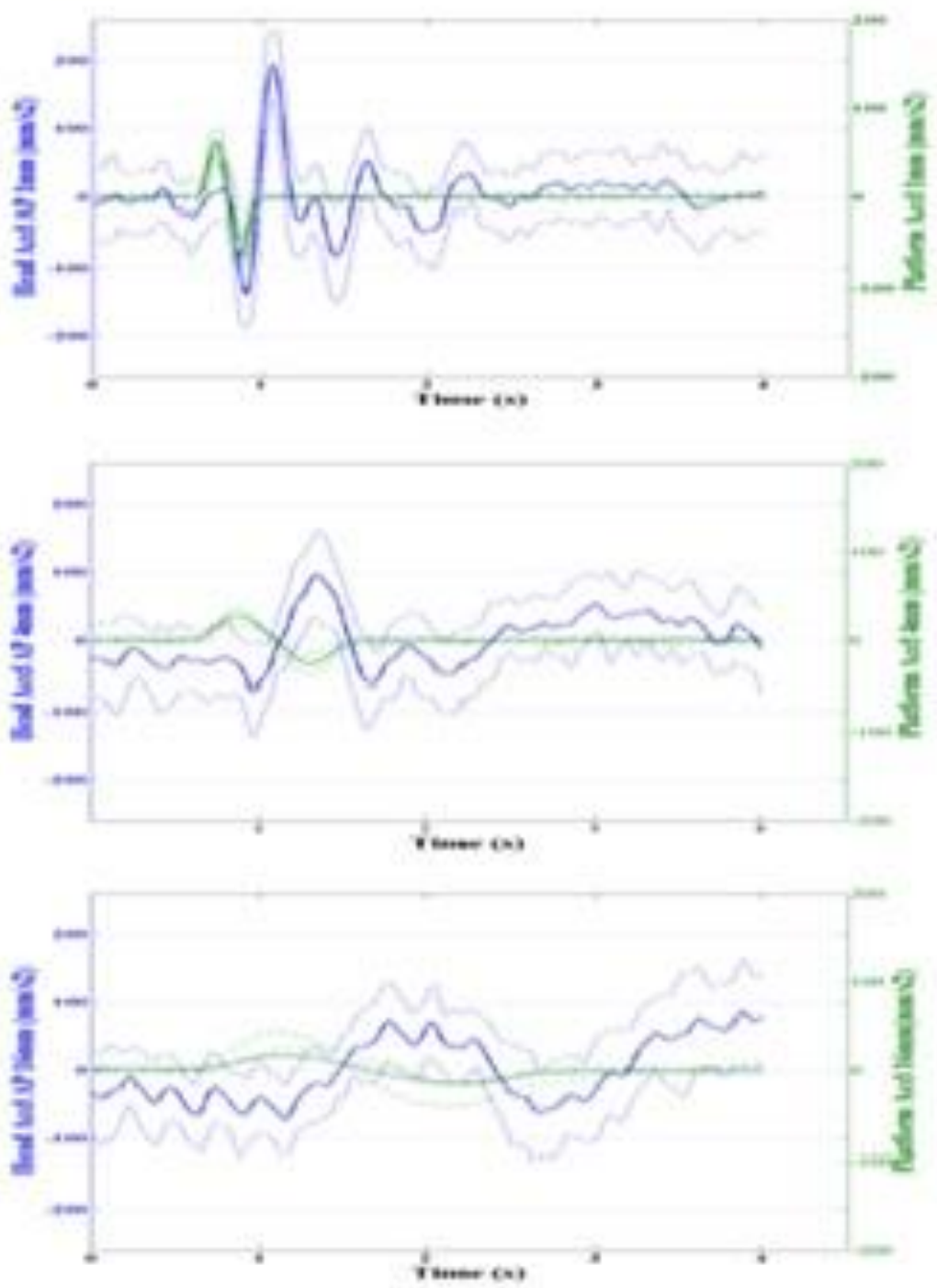

(a)

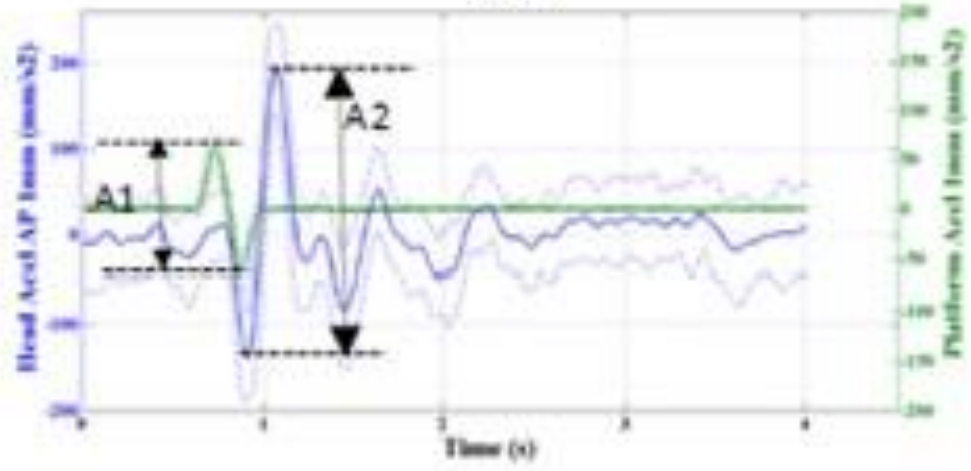

(b)

Fig. 3.a) Ensemble averaged (S \pm ) platform accelaration and ensemble averaged Head AP acceleration. b)Gain between platform acceleration as an input and Head AP acceleration as an output. 


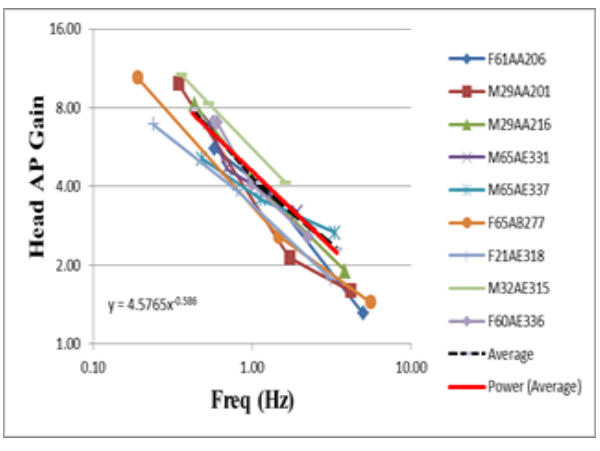

(a)

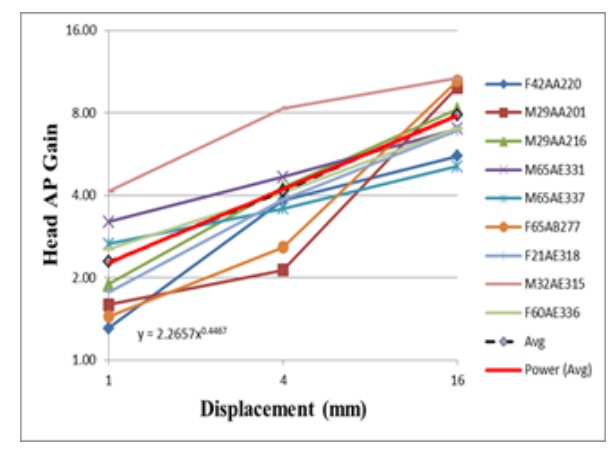

(b)

Fig. 4. a) Frequency dependence of the head AP gain in 9 healthy blindfolded standing subjects. b) Head AP gain versus different displacements $(1 \mathrm{~mm}, 4 \mathrm{~mm}$, and $16 \mathrm{~mm})$ in 9 healthy blindfolded standing subjects.

\subsection{Correlation between translation length and Head acceleration gain}

Figs. 4.a shows the head AP gain (between platform acceleration and head AP acceleration) versus different frequencies of perturbation $(1 \mathrm{~mm}, 4 \mathrm{~mm}, 16 \mathrm{~mm})$ in 9 healthy subjects. A negative power law exists between head AP gains and stimulus frequency. Fig. 4.b shows the head AP gain versus different perturbation displacements $(1 \mathrm{~mm}, 4 \mathrm{~mm}$, and $16 \mathrm{~mm})$ in 9 healthy subjects. A positive power law has been obviously observed between gain (Head AP) and stimulus displacements.

\section{DISCUSSION}

A Matlab based GUI (Graphical User Interface) was created to study the transference of acceleration from platform motion to subject's head. Different movement displacements $(1 \mathrm{~mm}$, $4 \mathrm{~mm}$, and $16 \mathrm{~mm}$ ) in $2 \mathrm{AFC}$ experiments helped us investigate the frequency dependence of subject's movement perception. In the $1 \mathrm{~mm}$ experiment, there were 2 differences between movement and non-movement head acceleration data. A signal with larger amplitude and lower frequency component was observed in the movement interval in the head acceleration data for $1 \mathrm{~mm}$. But in $4 \mathrm{~mm}$ and $16 \mathrm{~mm}$, only with lower frequency component was observed during the movement interval. In other words, at $4 \mathrm{~mm}$ and $16 \mathrm{~mm}$ there is not a tremendous difference in head acceleration amplitudes between movement and non-movement intervals.

Previous SLIP FALLS research revealed a negative power law relationship between the length of the short anterior translations and the subjects' Peak Acceleration Thresholds (PAT=38.90D0.56) [9]. We studied here the effect of the external perturbation frequency on the Head AP gain. This could account for the low PAT values at a $16 \mathrm{~mm}$ displacement. In other words, with increasing length of the short anterior translations (or the decreasing frequency of the platform acceleration), head AP gains increased. This could justify the low PAT values at the $16 \mathrm{~mm}$ displacement. But what does higher gain imply? Higher Head AP gain could imply more vestibular system input, possibly from the otoliths.

This study points out the sensitivity of Head AP gain to the frequency of the platform acceleration. An indirect relation was observed between the stimulus frequency and the gains. This could explain the results of Mah et al. [11] that reported a direct relationship between stimulus frequency and detection threshold (where an increase in frequency caused an increase in threshold). 
International Journal on Bioinformatics \& Biosciences (IJBB) Vol.6, No.3/4, December 2016

\section{REFERENCES}

[1] Gupta, A, "Effect of Stimulus Presentation Order on Two Alternative Forced Choice Psychophysical Responses in Standing Human Subjects: With or without Diabetic and Observed Differences in Physiological Cues," Dissertation, Louisiana Tech University, 2003.

[2] Bhatkar, V, "Determination of the Biopsychophysical Detection Threshold to Short postural," Dissertation, Clarkson University, 2010.

[3] Robinson, C. J., Purucker, M. C., \& Faulkner, L. W. "Design, control, and characterization of a sliding linear investigative platform for analyzing lower limb stability (SLIP-FALLS)”. IEEE Transactions on Rehabilitation Engineering, 6(3), 334-350

[4] Faulkner, L, "Psychophysical Detection Thresholds and Interactions Among Acceleration, Velocity and Displacement, Produced by Young Adults While Standing on a Platform Horizontally Translated Under Ultra-Low Vibration Conditions", PhD Dissertation in Rehabilitation Science, University of Pittsburgh, (2003).

[5] Bala subramaranian, V. "Postural Balance and Acceleration Threshold Detection for Anterior Horizontal Translation in Diabetic and non-Diabetic Elderly," Ph.D. dissertation in Biomedical Engineering, Louisiana Tech Univ. (2001).

[6] Robinson, CJ, Fulk, G, Mondal, S, "Comparing psychophysical detection thresholds to very short postural perturbations in mature adult with and without diabetes", Proc of the XIX Conf of the International Society for Posture and Gait Research Conference, Bologna, Italy, pg 176, (June 2009).

[7] Grant, P., and Haycock, B. "The effect of jerk and acceleration on the perception of motion Strength." AIAA Modeling and Simulation Conference (2006), pp. 303-313.

[8] Benson, A. J., Spencer, M. B. and Stott, J. R. R., "Thresholds for the Detection of the Direction of Whole-Body, Linear Movement in the Horizontal Plane”, Aviation Space and Environmental Medicine, Vol. 57, No. 11, 1986, pp. 1088-1096.

[9] Heerspink, H. M., Berkouwer, W. R., Stroosma, O., van Paassen, M. M., Mulder, M. and Mulder, J. A., "Evaluation ofVestibular Thresholds for Motion Detection in the SIMONA Research Simulator", AIAA Modeling and Simulation Technologies Conference and Exhibit, AIAA-2005-6502, Delft University of Technology, August 2005.

[10] Grant, P. R. and Haycock, B., "Effect of Jerk and Acceleration on the Perception of Motion Strength", Journal of Aircraft,Vol. 45, No. 4, 2008, pp. 1190-1197.

[11] Mah, R. W., Young, L. R., Steele, C. R. and Schubert, E. D., "Thresholds for the Perception of WholeBody Linear Sinusoidal Motion in the Horizontal Plane”, AIAA Flight Simulation Technologies Conference and Exhibit, AIAA 89-3273, NASA Ames Research Center, August 1989.

[12] Faulkner, L, "Psychophysical Detection Thresholds and Interactions Among Acceleration, Velocity and Displacement, Produced by Young Adults While Standing on a Platform Horizontally Translated Under Ultra-Low Vibration Conditions," Ph.D. Dissertation in Rehabilitation Science, University of Pittsburgh, 2003.

[13] Dong, X, "Determination of Psychophysical Detection Thresholds and Tibialis Anterior and Gastrocnemius EMGS Analysis During Subtle Anterior Postural Perturbations With Subjects Blindfolded Under Restrained Seated and Standing Condition," PhD Thesis, Clarkson University, 2013. 